$\mathrm{DTP} / 00 / 44$

July 2000

\title{
IMPACT OF THE W BOSON DECAY WIDTH ON PHOTON BREMSSTRAHLUNG ACCOMPANYING W PAIR PRODUCTION
}

\author{
A.P. Chapovsky ${ }^{1}$, V.A. Khoze ${ }^{1}$ and W.J. Stirling ${ }^{1,2}$ \\ 1 Department of Physics, University of Durham, Durham DH1 3LE, England \\ 2 Department of Mathematical Sciences, University of Durham, Durham DH1 3LE, England
}

\begin{abstract}
The bremsstrahlung spectrum of photons accompanying $W$ pair production depends on the decay width of the unstable $W$ bosons. The dependence arises from the interference between emission at different stages of the production and decay process. We present a quantitative discussion of this effect, and consider the implications for measurements at LEP2 and LC energies.
\end{abstract}

emails: A.P.Chapovsky@durham.ac.uk, V.A.Khoze@durham.ac.uk, W.J.Stirling@durham.ac.uk 


\section{Introduction}

Operating a future electron-positron linear collider (LC) in the LEP2 energy region but with much higher luminosity would allow a variety of precision tests of the electroweak sector to be performed, see for example Ref. [1]. For instance, a $100 \mathrm{fb}^{-1}$ scan of the $W^{+} W^{-}$threshold with longitudinally polarized electrons and positrons would offer an opportunity to measure the $W$ boson mass with an error of $6 \mathrm{MeV}$, [2], with negligible uncertainty from QCD interconnection effects, see for example Ref. [3]. Such a threshold scan could also potentially provide a precise measurement of the $W$ decay width, $\Gamma_{W}$.

At present, the most precise determination of $\Gamma_{W}$ comes from the indirect measurement at the Tevatron using the ratio of dilepton $Z$ and $W$ events [四]:

$$
\Gamma_{W}(\mathrm{CDF}+\mathrm{D} 0, \text { indirect })=2.171 \pm 0.027 \text { (stat.) } \pm 0.056 \text { (sys.) } \mathrm{GeV}
$$

i.e. with an overall precision of approximately $60 \mathrm{MeV}$. However it is very important to perform a direct determination of this key parameter of the Standard Model as well. The accuracy of the direct measurement of the $W$ width is still not very high. Recently the CDF collaboration at the Tevatron have reported the value [5]:

$$
\Gamma_{W}(\mathrm{CDF}, \text { direct) }=2.055 \pm 0.100 \text { (stat.) } \pm 0.075 \text { (sys.) } \mathrm{GeV}
$$

from measurements of the transverse mass spectrum in leptonic $W$ decays. Finally, the LEP experiments have made a preliminary measurement of the $W$ width from the line shape in $W$ pair production [6]:

$$
\left.\Gamma_{W}(\mathrm{LEP} 2, \text { direct })=2.19 \pm 0.15 \text { (stat. }+ \text { sys. }\right) \mathrm{GeV}
$$

Another related method, not yet exploited, would be to perform a precision scan of the $W W$ cross section in the threshold region, for example at a future linear collider.

In this paper we focus on another method which is based on previous studies in Ref. [7]. This exploits the high sensitivity of soft photon radiation in pair production of $W$ bosons in $e^{+} e^{-}$ collisions to the $W$ width. Since the event rate is $\mathcal{O}(\alpha)$ relative to the total $W W$ cross section, this method is potentially limited by statistics. However it has the advantage of avoiding problems such as the effect of beamsstrahlung or beam energy spread on line shapes. Whether it will ultimately be statistically competitive with a threshold scan in the precise determination of $\Gamma_{W}$ will require a dedicated analysis, which is beyond the scope of our studies here. However, our results do suggest that such further investigation would certainly be worthwhile.

The paper is organized as follows. In the next section we discuss the overall structure of the $e^{+} e^{-} \rightarrow W^{+} W^{-} \gamma$ differential cross section, emphasizing the various energy regimes for the photon radiation. We present analytic results for the cross section in the soft-photon limit, which allows us to identify the factorizable and non-factorizable contributions. In Section 3 we discuss the various ways of enhancing the non-factorizable contributions, which contain the bulk of the $\Gamma_{W}$ dependence, by imposing angular cuts on the final-state particles. We illustrate our results by numerical calculations, considering the various possible leptonic and hadronic decay channels. We also mention briefly the analogous results for the $\gamma \gamma \rightarrow W^{+} W^{-} \gamma$ process. Section 4 contains our conclusions. 


\section{Bremsstrahlung radiation pattern in $W^{+} W^{-}$produc- tion}

The general formalism for calculating the soft radiation pattern in processes involving the production and decay of unstable particles can be found in Refs. [8, 7, 9, 10, 11]. It is well known that heavy unstable particles such as the $W$ boson can radiate before and after their decay. The relative intensity of the two contributions, and consequently the overall structure of the radiation pattern, depends sensitively on the relative size of the emission time-scale and the particle lifetime, see e.g. [7, 8]. In particular, in the second reference in [7] one can find a semi-classical explanation of how the radiation pattern allows the relative distance between the $W$-boson decay vertices to be probed."

We begin by recalling the main properties of the differential distribution for the radiation of a soft photon with momentum $k^{\mu}$ in the process

$$
e^{+}\left(q_{1}\right) e^{-}\left(q_{2}\right) \rightarrow W^{+}\left(p_{1}\right) W^{-}\left(p_{2}\right) \rightarrow 4 \text { fermions }\left(p_{3}, p_{3}^{\prime}, p_{4}, p_{4}^{\prime}\right)[+\gamma(k)] .
$$

It is well known that when unstable particles are produced one is forced to perform a Dyson resummation, which leads to the regularization of the singularities in the propagators $1 /\left(M_{1,2}^{2}-\right.$ $\left.M_{W}^{2}\right) \rightarrow 1 / D_{1,2}$, where $D_{1,2}=M_{1,2}^{2}-M_{W}^{2}+i M_{W} \Gamma_{W}$, and $M_{1,2}$ are the invariant masses of the $W$ bosons. However such resummation leads in general to the breaking of gauge invariance through higher-order contributions picked up by Dyson resummation. Thus this standard perturbative approach does not produce an acceptable gauge independent answer. The problem can be avoided by working in the so-called 'pole-scheme' [13]. The physical picture behind the polescheme is as follows. Any process involving unstable particles can be viewed as a consequence of several subprocesses: production, which is a hard process with a short time-scale $\mathcal{O}\left(1 / M_{W}\right)$; propagation over a typically larger time $\mathcal{O}\left(1 / \Gamma_{W}\right)$; and decay, which is again a hard process with a time scale $\mathcal{O}\left(1 / M_{W}\right)$. Technically, in the perturbative expansion gauge invariance is guaranteed only order by order. Dyson resummation mixes different orders of the perturbative expansion, and thus breaks gauge invariance. In order to restore it one has to re-expand the amplitudes again in some physical parameter other than the coupling constant, in a way that does not produce singularities. An appropriate small parameter is $\Gamma_{W} / M_{W}$. It is constructed as a ratio of two physical scales: the scale of production and decay, $M_{W}$, and the scale of propagation, $\Gamma_{W}$. It should be noted that this is a somewhat simplified picture, since sometimes there are additional small parameters present in the problem (like the relative velocity, $\beta$, close to threshold, or $M_{W}^{2} / s$ at ultra-relativistic energies, etc.). Then the above mentioned estimates may change, but the arguments remain similar.

From the above considerations one can estimate the accuracy of calculations performed in the pole-scheme. When examining the process (四) one distinguishes three energy domains classified by the distance in energy from threshold, $\Delta E=\sqrt{s}-2 M_{W}$, compared to the relevant scales of the process, $\Gamma_{W}$ and $M_{W}$ :

\footnotetext{
${ }^{1}$ This phenomenon resembles an old idea [12] to use soft-photon radiation for measuring the time delay in nuclear reactions.
} 
- Relativistic region, $\Delta E \sim M_{W}$, where the accuracy is $\mathcal{O}\left(\Gamma_{W} / M_{W}\right)$.

- 'Far-from-threshold' region, $\Gamma_{W} \ll \Delta E \ll M_{W}$, where the accuracy is $\mathcal{O}\left(\Gamma_{W} / \Delta E\right)$.

- Threshold region, $\Delta E \sim \Gamma_{W}$, where the accuracy is $\mathcal{O}(1)$, and the pole-scheme expansion breaks down.

The pole-scheme approach to processes involving unstable particles has been used to calculate the full $\mathcal{O}(\alpha)$ correction to the pair production of $W$ bosons in $e^{+} e^{-}$collisions [9, 10]. In this paper we use the results of Refs. [7, 9] as a basis for the calculations. Because of the way the pole-scheme is constructed, one can classify all the radiative corrections into two types: factorizable, which act inside separate hard subprocesses (production and decay); and nonfactorizable, which interconnect various hard subprocesses. Here we will concentrate on the real photon radiation from $W$-pair production in the LEP2/LC energy region $170-500 \mathrm{GeV}$. Again, there are three regimes for photon radiation.

- Hard photon radiation, $\omega \sim M_{W}$, when the photon wavelength is of the same order as the hard process time-scale. The photon can be assigned to one of the hard subprocesses. Alternatively one can say that the photons radiated from different stages of the process do not interfere with each other. The radiation is exclusively factorizable.

- Soft radiation, $\omega \ll \Gamma_{W}$, when the photon wavelength is much larger than the propagation distance. In this case the photons cannot distinguish the details of the process and are radiated coherently from all stages of the process. Both factorizable and non-factorizable contributions are important.

- Semi-soft radiation, $\omega \sim \Gamma_{W}$, when the photon wavelength is of the same order as the distance between the $W$ decay vertices. In this case both factorizable and non-factorizable contributions are important. However photons are not radiated coherently from all stages of the process.

From this classification one can see that at $\omega \sim \Gamma_{W}$ there is a transition from a regime in which various subprocesses do not interfere with each other to a regime in which the photon does not distinguish details of the process. This is this transition that is of interest to us in this paper, since it is where the photon spectrum has maximum sensitivity to $\Gamma_{W}$.

Note that when $\omega \sim \Gamma_{W}$ the photon is soft with respect to the hard scale of the process, $\omega \ll M_{W}$, but not with respect to the soft scale of the process, $\Gamma_{W}$. As a consequence the cross section has certain factorization properties. The hard ( $M_{W}$-scale) part of the amplitude factorizes just as in the conventional soft-photon approximation, but the soft $\left(\Gamma_{W}\right.$-scale) part does not always factorize in the conventional way. This is why we call photons with energy $\omega \sim \Gamma_{W}$ 'semi-soft', rather than simply 'soft'. Making use of the factorization properties, the complete radiation distribution in this semi-soft regime can be written as an interference of semi-soft currents with the hard parts of the amplitudes in the following way:

$$
d \sigma=-d \sigma_{\text {Born }} \frac{d \vec{k}}{(2 \pi)^{3} 2 k_{0}}\left[2 \operatorname{Re}\left(\mathcal{I}_{0} \cdot \mathcal{I}_{+}^{*}+\mathcal{I}_{0} \cdot \mathcal{I}_{-}^{*}+\mathcal{I}_{+} \cdot \mathcal{I}_{-}^{*}\right)+\mathcal{I}_{0} \cdot \mathcal{I}_{0}^{*}+\mathcal{I}_{+} \cdot \mathcal{I}_{+}^{*}+\mathcal{I}_{-} \cdot \mathcal{I}_{-}^{*}\right]
$$


Here $d \sigma_{\text {Born }}$ is the Born cross section in the pole approximation. The currents $\mathcal{I}_{0}$ and $\mathcal{I}_{ \pm}$ correspond to the radiation from the production and decay stages respectively. The first three terms are non-factorizable contributions, consisting of final-final, $\mathcal{I}_{+} \mathcal{I}_{-}^{*}$, and initial-final, $\mathcal{I}_{0} \mathcal{I}_{+}^{*}+$ $\mathcal{I}_{0} \mathcal{I}_{-}^{*}$, state interferences. The last three terms are the factorizable contributions corresponding to the production and decay parts. The gauge-invariant semi-soft currents $\mathcal{I}_{0}$ and $\mathcal{I}_{ \pm}$are given by

$$
\begin{aligned}
& \mathcal{I}_{0}^{\mu}=+e\left[\frac{p_{1}^{\mu}}{k \cdot p_{1}}-\frac{p_{2}^{\mu}}{k \cdot p_{2}}-\frac{q_{1}^{\mu}}{k \cdot q_{1}}+\frac{q_{2}^{\mu}}{k \cdot q_{2}}\right], \\
& \mathcal{I}_{+}^{\mu}=-e\left[\frac{p_{1}^{\mu}}{k \cdot p_{1}}+Q_{f_{3}} \frac{p_{3}^{\mu}}{k \cdot p_{3}}-Q_{f_{3}^{\prime}} \frac{p_{3}^{\prime}{ }^{\mu}}{k \cdot p_{3}^{\prime}}\right] \frac{D_{1}}{D_{1}+2 k \cdot p_{1}}, \\
& \mathcal{I}_{-}^{\mu}=+e\left[\frac{p_{2}^{\mu}}{k \cdot p_{2}}+Q_{f_{4}} \frac{p_{4}^{\mu}}{k \cdot p_{4}}-Q_{f_{4}^{\prime}} \frac{p_{4}^{\prime}{ }^{\mu}}{k \cdot p_{4}^{\prime}}\right] \frac{D_{2}}{D_{2}+2 k \cdot p_{2}} .
\end{aligned}
$$

The factors $Q_{f}, Q_{f^{\prime}}$ are the electric charges of the final-state fermions, with $Q_{f}-Q_{f^{\prime}}=-1$. Recall that the integration over the invariant masses of the unstable particles eliminates the pre-factors $D_{1,2} /\left(D_{1,2}+2 k \cdot p_{1,2}\right)$ in the factorizable terms. In this case the semi-soft currents become the usual soft-photon ones, and factorization takes place with respect to both scales of the process, hence the name 'factorizable'. In the non-factorizable contributions, however, non-trivial pre-factors survive, and complete factorization with respect to both scales does not take place. An important consequence of this non-factorization is that for hard photons the non-factorizable contribution is suppressed because of the photon energy dependence in the pre-factors, see also Refs. [7, 8, 14]. Thus non-factorizable contributions are important only for soft and semi-soft photons.

The qualitative picture described above is illustrated quantitatively in Fig. 1, which shows the photon energy spectrum, $1 / \sigma_{\text {Born }} \omega d \sigma / d \omega$, is shown as a function of the photon energy, $\omega$, in the semi-soft region. If In this example the $e^{+} e^{-}$CMS energy is $\sqrt{s}=184 \mathrm{GeV}$ and a purely leptonic $\left(\mu^{+} \nu_{\mu}\right)\left(\tau^{-} \bar{\nu}_{\tau}\right)$ final state is chosen. A photon 'isolation' cut is also applied. This requires that in the CMS frame the direction of the radiated photon is separated by at least $50^{\circ}$ from the directions of all the experimentally observed charged particles (i.e. the initial-state $e^{ \pm}$ and the final-state $\mu$ and $\tau$ leptons). By imposing these 'no-flight' zones around the charged particles we avoid the quasi-collinear-singularities inherent in the currents in (6).

We can see from Fig. 1 that the (negative) non-factorizable contribution to the cross section is indeed suppressed for hard photons, with the damping occurring in the semi-soft regime of the photon energy, $\omega \sim \Gamma_{W} \approx 2 \mathrm{GeV}$. In the same photon energy region the dependence of the factorizable contribution on $\omega$ is practically flat. This leads to a peaking behaviour of the complete spectrum in the semi-soft region, $\omega \sim \Gamma_{W}$. Thus by comparing the measured photon bremsstrahlung distribution in this region with the theoretical prediction regarded as a function of $\Gamma_{W}$, one can in principle determine the $W$-boson width, as advocated in Ref. [7].

\footnotetext{
${ }^{2}$ Here and below we use the results and parameter values of Ref. [9] for numerical calculations. In particular we use the Standard Model $W$-boson width $\Gamma_{W}=2.082 \mathrm{GeV}$.

${ }^{3}$ In practice, the collinear singularities are regulated by non-zero fermion masses, see below.
} 


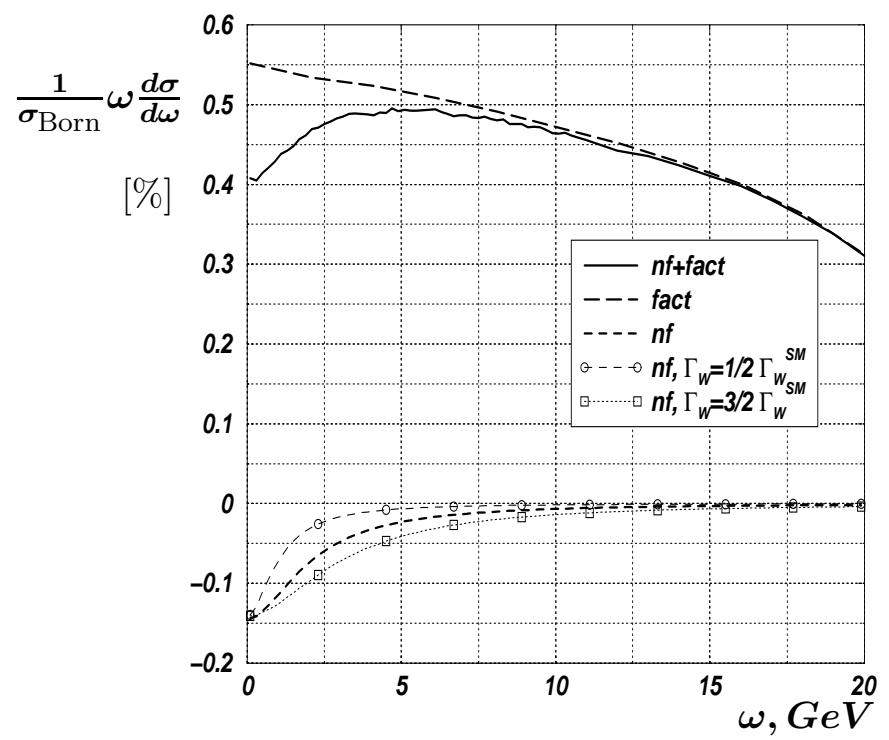

Figure 1: The photon energy distribution, $\frac{1}{\sigma_{\text {Born }}} \omega \frac{d \sigma}{d \omega}$, normalized to the Born cross-section, $\sigma_{\text {Born }} \approx 0.22 \mathrm{pb}$, as a function of the photon energy, $\omega$, in the semi-soft regime. The final state is $\left(\mu^{+} \nu_{\mu}\right)\left(\tau^{-} \bar{\nu}_{\tau}\right)$ and the $e^{+} e^{-}$CMS energy is $\sqrt{s}=184 \mathrm{GeV}$. The photon isolation cut, which restricts the photon to be separated by at least $50^{\circ}$ from all the charged fermions, is applied. Also shown is the non-factorizable contribution calculated for various values of the width, $\Gamma_{W}=(1 / 2 ; 1 ; 3 / 2) \Gamma_{W}^{S M}$, illustrating strong width dependence.

In order to gain some quantitative insight on how the method may work in practice, two issues are important:

- How pronounced is the shape of the relevant part of the photon spectrum? In other words, how large is the interesting (strongly $\Gamma_{W}$ dependent, see Fig. 1) non-factorizable contribution with respect to the factorizable contribution? The relevant parameter here is

$$
\alpha(\sqrt{s}, \mathrm{cuts})=\left.\frac{\left(d \sigma_{\mathrm{nf}} / d \omega\right)}{\left(d \sigma_{\text {fact }} / d \omega\right)}\right|_{\omega \rightarrow 0},
$$

which depends on the system of cuts chosen and the collider energy, $\sqrt{s}$.

- How large is the statistics for a particular choice of cuts? The relevant parameter is the corresponding Born cross section restricted by a particular system of cuts, $d \sigma_{\operatorname{Born}}(\sqrt{s}$, cut).

To illustrate how the ratio of non-factorizable to factorizable radiation, $\alpha$ (no cuts, $\sqrt{s}$ ), is influenced by the photon isolation cuts, we show in Fig. 2 the ratio as a function of the CMS energy, $\sqrt{s}$, with and without cuts. In order to use a logarithmic scale we plot the absolute values of the ratios, and indicate their sign in the legend of the plot. Here and in what follows we label all quantities by two letters, which specify the decay channel of each of the $W^{\prime}$ 's, $L$ for leptonic and $H$ for hadronic, and a subscript, which specifies the system of cuts applied to the kinematics. In this case we consider a purely leptonic final state (in this example $\mu^{+} \tau^{-}$), thus 


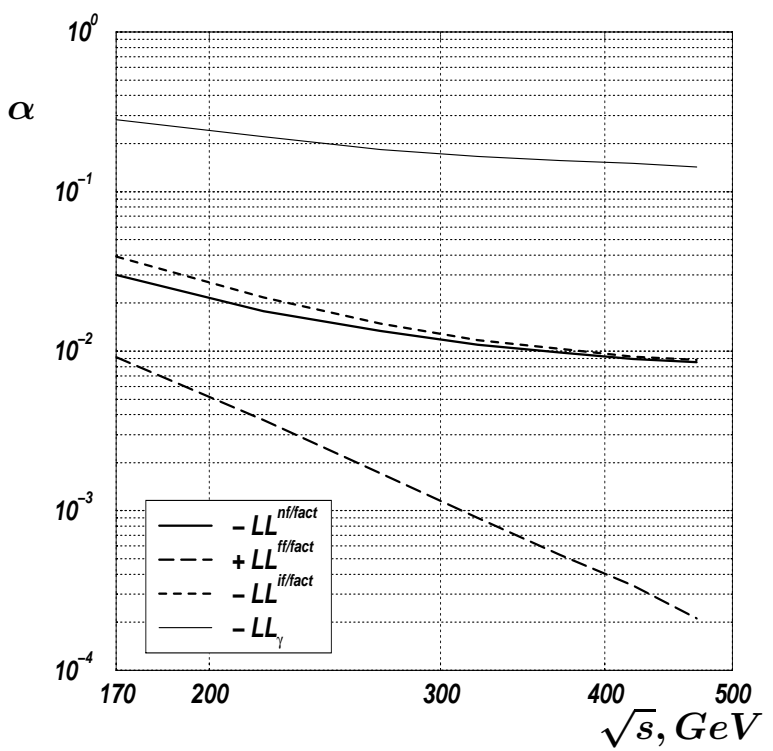

Figure 2: The absolute value of the ratio of non-factorizable and factorizable contributions to the cross section, $\alpha$, for the leptonic $\mu \tau$ final state, as a function of the CMS energy with $\left(L L_{\gamma}\right)$ and without $\left(L L^{\mathrm{nf} / \mathrm{fact}}\right)$ photon isolation cuts $\left(>50^{\circ}\right.$ from all charged particles). The sign of the ratio is show in the legend of the plot. In the case of no cuts, the initial-final, $L L^{\text {if } / \text { fact }}$, and final-final, $L L^{\mathrm{ff} / \mathrm{fact}}$, interferences are shown separately.

the label is $L L$. The factorizable radiation for the 'no-cut' case depends on the masses of the charged fermions through collinear logarithms.

In Fig. 2, in addition to the combined factorizable/non-factorizable effect, $L L^{\text {nf/fact }}$, we show separately the initial-final, $L L^{\text {if/fact }}$, and final-final, $L L^{\mathrm{ff} / \mathrm{fact}}$, state ratios. Note that they have opposite signs.t One can see from the figure that the final-final part of the non-factorizable correction scales as $\alpha \sim E^{-4}$ with the CMS energy. In fact the power-counting arguments of Ref. [15] are applicable to the parameter $\alpha$, with a small modification due to the fixed rather than integrated photon energy which does not however change the result. For the initial-final part of the interference the energy scaling is different: $\alpha \sim E^{-2}$. One can also see that initialfinal state interference dominates the non-factorizable effects at high energies, f and thus the complete non-factorizable radiation contribution also scales as $E^{-2}$. If one does not apply any cuts, the ratio of non-factorizable to factorizable contributions is small, below $3 \%$. This is mainly due to the enhancement of the collinear logarithms in the factorizable part of the radiation. However if one keeps the photon well separated from the charged particles, and thus well away from the collinear regions, as in the $L L_{\gamma}$ ratio in Fig. 2 then the collinear logarithms are suppressed and the ratio increases considerably. For example, in Fig. $2 \alpha \approx 10-30 \%$ for the $L L_{\gamma}>50^{\circ}$ cut, depending on the collider energy.

\footnotetext{
${ }^{4}$ This agrees with the observations of Ref. 8 where gluon radiation in $e^{+} e^{-} \rightarrow t \bar{t} \rightarrow b W^{+} \bar{b} W^{-}$was discussed.

${ }^{5}$ This is again in agreement with observations of Ref. [8].
} 


\section{Enhancement mechanisms for different external states}

In Ref. [7] two processes were considered in detail: gluon radiation in $e^{+} e^{-} \rightarrow t \bar{t}$ and photon radiation in $\gamma \gamma \rightarrow W^{+} W^{-}$. Both cases were considered for collision energies close to threshold. The process we are interested in, (4), differs from the studies of [7] in two respects.

First, we consider higher collision energies, which are experimentally more relevant. Moreover the pole expansion, which we use in our calculations, does not apply at threshold. On the other hand, as we have already seen, at higher-energies non-factorizable effects become relatively small and therefore the sensitivity to $\Gamma_{W}$ is less [11, 15]. Without any cuts, the non-factorizable corrections, (5), to distributions inclusive with respect to angles scale as $E^{-2}$ (initial-final state interference) or $E^{-4}$ (final-final state interference) relative to the Born cross section. As a result of this scaling behaviour, at $\sqrt{s}=184 \mathrm{GeV}$ the ratio of non-factorizable to factorizable contributions to the photon spectrum is $\mathcal{O}(1 \%)$ or smaller. The main objective of our studies is to enhance the non-factorizable effects by applying angular cuts.

A second difference with the study of Ref. [7] is that there the effects of initial state radiation were not fully considered. In terms of Eq. (5), the analysis of Ref. [7] was concerned with only one of the non-factorizable effects, from final-final state interference. As we have already noted, for $e^{+} e^{-} \rightarrow W^{+} W^{-}+\gamma$ the situation is more complicated, with three interference contributions: two initial-final and one final-final state.

In Ref. [7] two ways to enhance the non-factorizable effects were proposed. First, it was suggested that certain angular asymmetry properties of initial-final state interference, absent in final-final state interference, could be used to construct observables to which initial-final state interference does not contribute. Moreover, in [7] an observable was constructed which has no contributions from factorizable radiation, using the fact that the factorizable correction does not depend on the angles of the produced particles, at least at threshold (see Ref. [7] for more details).

Unfortunately, the construction of such observables is impractical. Because of $t$-channel neutrino exchange, there are always spin-charge correlations present, even in the threshold Born cross section. Initial-final spin correlations induced by the $W$ propagators lead to an asymmetry in the factorizable part of the radiation, as well as in the final-final state interference contributions. Therefore the method proposed in Ref. [7] does not appear to be workable. Note that this effect originates in the $(v-a)$ structure of the charged weak current. The Born DPA cross section does not violate $P$-parity because only pole residues are calculated. However the type of helicity-charge correlation described above does survive. Technically, anti-symmetric tensors, $\epsilon_{\mu \nu \rho \sigma}$, induced by the axial current do not contribute linearly to the matrix element (no $P$-violation), but only quadratically, via the interference of axial contributions from various stages of the process (the helicity-charge asymmetry).

\footnotetext{
${ }^{6}$ Note that in $W$ pair production in $\gamma \gamma$ collisions the asymmetry in the threshold Born cross section is absent, because there is no $(v-a)$ structure at the production stage. Therefore, all the results of Ref. [7] remain valid for the $\gamma \gamma \rightarrow W^{+} W^{-}$case. The asymmetry is also absent in $e^{+} e^{-} \rightarrow Z Z$ production, because of Bose symmetry. On the other hand the asymmetry will be present, even without the $(v-a)$ coupling of initial-state fermions
} 
Another idea discussed in [7] was based on the 'angular ordering' effect. During the last two decades such angular ordering effects have been intensively discussed in the context of the QCD cascades, see for example [16]. The phenomenon itself has been well known for QED in cosmic ray physics from the middle of the 1950s as the so-called Chudakov effect [17]. To recall the physics of angular ordering, we consider the radiation pattern of soft photons produced by a relativistic $e^{+} e^{-}$pair. If we split the radiation into pieces associated with the $e^{-}$and $e^{+}$, and then integrate over the azimuthal angle about, say, the $e^{-}$direction, the $e^{-}$contribution vanishes for polar angles greater than the $e^{+} e^{-}$opening angle. In particular this implies that the radiation vanishes for collinear $e^{+}$and $e^{-}$. In other words, for such a configuration the emitted photon probes only the total electric charge of the $e^{+} e^{-}$pair, which is zero. The suppression of radiation is caused by the (destructive) interference between the emission off the $e^{-}$and $e^{+}$, see the second reference in [7] for more details. Because in the present context it is the $W$ lifetime that controls this interference pattern, we expect to observe angular ordering behaviour (or not) according to the size of the ratio $\omega / \Gamma_{W}$. It is therefore clear that the largest effect of non-factorizable radiation relative to factorizable radiation will correspond to the case of collinear oppositely charged particles. In that case factorizable radiation is as important as non-factorizable radiation.

In the case of $W$ pair production with $\mu \nu_{\mu} \tau \nu_{\tau}$ decay in the threshold region there are four radiating charged particles: two initial state fermions, $e^{ \pm}$, and two final state fermions, $\mu^{+}, \tau^{-}$. Corresponding to this there are three non-factorizable interferences: two initial-final, and one final-final state interference. Clearly it is impossible to generate a large effect from all three interferences simultaneously. Indeed, if the $e^{+}$and $\mu^{+}$are collinear and the $e^{-}$and $\tau^{-}$are collinear, then the $\mu^{+}$and $\tau^{-}$are anti-collinear. Far above threshold, the directions of the $W$-boson momenta start to play a role as well. Thus in general one has many cases when some oppositely charged particles are collinear and others are not, leading to a non-trivial interplay between the various interference terms in (5). Rather than choose particular fixed configurations, for which the statistics will be small, it is more efficient to look for angular cuts (no-flight zones) on the various particles such that the interesting (i.e. most $\Gamma_{W}$ dependent) events are favoured but not overly restricted. We shall not in the present study make any serious attempt to optimize these cuts; rather we will present some illustrative examples pending more detailed Monte Carlo analyses.

In summary, angular cuts (no-flight zones) will be applied to the final state particles (leptons and quarks) and the photon in order to maximize the angular ordering effect, and thus the sensitivity of the photon spectrum to the $W$ width. As explained above, the basic idea is to keep oppositely charged particles quasi-collinear, and the photon as far from them as possible. There is an additional requirement motivated by detector considerations. The final state particles should not be too close to the beam direction otherwise the event cannot be unambiguously identified as $W^{+} W^{-} \gamma$. We therefore require all final state particles to be produced at polar

(as in QED for example), if the initial-state fermions are polarized.

7 Note that due to the celebrated Low-Kroll-Barnett soft bremsstrahlung theorem [18] the non-classical short-distance-induced corrections to the angular ordering behaviour arise only on the level of quadratic in $\omega / M$ terms, see Ref. [19]. 
angles greater than $5^{\circ}$ from the beam direction.

\subsection{Leptonic-leptonic final state}

The simplest case to analyse is when both $W^{\prime}$ 's decay leptonically:

$$
e^{+}\left(q_{1}\right)+e^{-}\left(q_{2}\right) \rightarrow \mu^{+}\left(p_{3}\right)+\tau^{-}\left(p_{4}\right)+2 \nu+\gamma(k)
$$

The first topology we will consider is when the two final-state charged particles are close to the beam direction. In this case the initial-final state interference gives a large effect. In addition, the photon should be far from the beam direction:

$$
\angle\left(q_{1,2} k\right)>50^{\circ}
$$

The charged final-state leptons with momenta $p_{3}$ and $p_{4}$ can each be either collinear to the initial-state positron or to the electron. 'Collinear' is here defined as being produced with polar angle between $5^{\circ}$ and $10^{\circ}$ with respect to the beam direction:

$$
\angle\left(q_{1} p_{3,4}\right) \in\left(5^{\circ}, 10^{\circ}\right), \quad \text { or } \quad \angle\left(q_{2} p_{3,4}\right) \in\left(5^{\circ}, 10^{\circ}\right)
$$

We therefore have four possible cases, which we label

$$
L L_{++}, \quad L L_{+-}, \quad L L_{-+}, \quad L L_{\times \times}
$$

corresponding to $\left(p_{3} \| q_{1}\right.$ and $\left.p_{4} \| q_{1}\right),\left(p_{3} \| q_{1}\right.$ and $\left.p_{4} \| q_{2}\right)$, and $\left(p_{3} \| q_{2}\right.$ and $\left.p_{4} \| q_{1}\right)$ correspondingly. $L L$ refers to the fact that both $W$ bosons decay leptonically. In the last case $L L_{\times \times}$we demand only that the final-state leptons are collinear with the electron and positron beams, without tracing the electric charge flow.

The second class of cuts we will consider is when two final-state particles are quasi-collinear. In this case it is the final-final state interference that produces a large effect. We first demand that all final-state particles are observable

$$
\angle\left(q_{1,2} p_{3,4}\right)>5^{\circ},
$$

and then that the final-state charged particles are collinear

$$
\angle\left(p_{3} p_{4}\right)<10^{\circ}
$$

and the photon is far from all charged particle directions

$$
\angle\left(p_{3,4} k\right)>50^{\circ}, \quad \angle\left(q_{1,2} k\right)>50^{\circ} .
$$

Here there is only one possible case, which we label

$$
L L_{\mathrm{f}}
$$



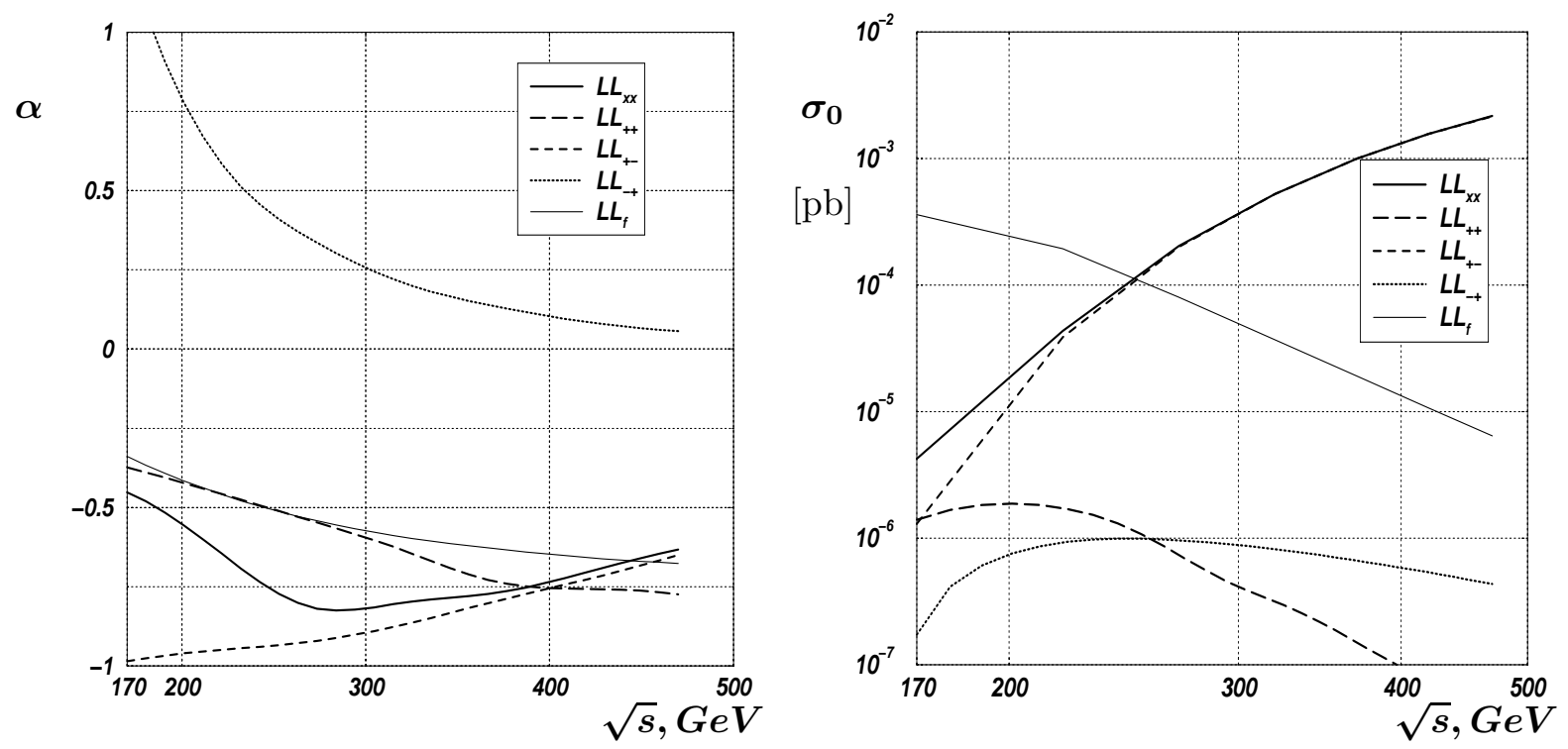

Figure 3: Ratio of the non-factorizable and factorizable parts of the photon radiation, $\alpha$, and the Born cross section, $\sigma_{\text {Born }}$, as a function of the CMS energy and the system of cuts. Purely leptonic final state only.

The optimization parameters $\alpha(\sqrt{s}$, cuts $)$ and $\sigma_{\text {Born }}(\sqrt{s}$, cuts $)$ are shown in Fig. 3 as functions of the CMS energy, $\sqrt{s}$, for all five possible leptonic cuts. We see that at low energies the most pronounced shape of the photon spectrum is achieved for the $L L_{-+}$case, i.e. $\mu^{+}$collinear with incoming $e^{-}$and $\tau^{-}$collinear with incoming $e^{+}$. Then the ratio of the non-factorizable to and factorizable contributions is positive and can even exceed 1, in the lower energy domain. However, the Born cross-section for this set of cuts is very small, which makes this case statistically disadvantageous. At high energies, the outgoing fermion (antifermion) prefers to follow the direction of the incoming fermion (antifermion), and hence both the $L L_{+-}$and $L L_{\times \times}$configurations have large Born cross sections, $\sigma_{\text {Born }}$. In terms of the shape parameter, the $L L_{+-}$ cut is as good as $L L_{\times \times}$. At lower energies, however, $L L_{+-}$becomes more advantageous in terms of shape, but less advantageous in terms of statistics. In fact, referring back to Fig. 2, we see that the original $L L_{\gamma}$-cut is as good in terms of shape as $L L_{\times \times}$at low energy, but much better statistically since it corresponds to a much larger angular acceptance for the final-state charged particles. At higher energies it is still as good in terms of shape, but becomes statistically very poor.

The conclusion is that depending on the energy and statistics available, one can choose different systems of cuts as the preferred ones. There is clearly no unique 'best cut' for all energies and all statistics.

\footnotetext{
${ }^{8}$ We are grateful to G. Wilson for clarification of various experimental issues related to $W$ studies at a future linear collider.
} 


\subsection{Leptonic-hadronic final state}

We next consider the case when the $W^{+}$decays leptonically and the $W^{-}$decays hadronically. There is one charged lepton and two jets present in the final state:

$$
e^{+}\left(q_{1}\right)+e^{-}\left(q_{2}\right) \rightarrow \mu^{+}\left(p_{3}\right)+q\left(p_{4}\right)+\bar{q}^{\prime}\left(p_{4}^{\prime}\right)+\nu+\gamma(k)
$$

We again start with the case when the charged primary fermions are close to the beam directions. Just as in the lepton-lepton case, we demand that the photon is far from the beams, $\angle\left(q_{1,2} k\right)>50^{\circ}$ and the final-state lepton with momenta $p_{3}$ is either collinear to the initial-state positron or electron, $\angle\left(q_{1} p_{3}\right) \in\left(5^{\circ}, 10^{\circ}\right)$ or $\angle\left(q_{2} p_{3}\right) \in\left(5^{\circ}, 10^{\circ}\right)$. The two quarks with momenta $p_{4}$ and $p_{4}^{\prime}$ should also be collinear to the initial-state particles:

$$
\angle\left(q_{1} p_{4}\right) \in\left(5^{\circ}, 20^{\circ}\right), \quad \text { or } \quad \angle\left(q_{2} p_{4}\right) \in\left(5^{\circ}, 20^{\circ}\right)
$$

and

$$
\angle\left(q_{1} p_{4}^{\prime}\right) \in\left(5^{\circ}, 20^{\circ}\right), \quad \text { or } \quad \angle\left(q_{2} p_{4}^{\prime}\right) \in\left(5^{\circ}, 20^{\circ}\right) .
$$

An important difference here is that one cannot measure the charge of the jet experimentally. Thus in general the following combinations are available:

$$
\begin{gathered}
L H_{+(20)}, \quad L H_{+(02)}, \quad L H_{-(20)}, \quad L H_{-(02)}, \\
L H_{+(11)}, \quad L H_{-(11)}, L H_{+(\times \times)}, L H_{-(\times \times)}, L H_{\times(\times \times)},
\end{gathered}
$$

where $L H$ denotes the leptonic-hadronic final state. The first subscript indicates the direction of the lepton with respect to the positron momentum, and the two subscripts in parenthesis indicate the number of jets collinear with the positron and electron. For example, $L H_{+(20)}$ means that the final-state lepton is collinear with the positron, as are both jets. $L H_{-(\times \times)}$means that the final-state lepton is collinear with the electron, and the two jets are collinear to either the positron or electron. Thus, in general, the number of different cases is rather large compared to the purely leptonic final state. For the energies we are interested in, $\sqrt{s}=170-500 \mathrm{GeV}$, however, the situation simplifies somewhat, because the kinematics are such that the two jets coming from the decay of the $W$ boson cannot in fact satisfy the collinearity selection criterion. Thus

$$
L H_{+(20)}=L H_{+(02)}=L H_{-(20)}=L H_{-(02)}=0 .
$$

The following cases survive

$$
L H_{+(11)}=L H_{+(\times \times)} \equiv L H_{+\times}, \quad L H_{-(11)}=L H_{-(\times \times)} \equiv L H_{-\times}, \quad L H_{\times(\times \times)} \equiv L H_{\times \times},
$$

where we have introduced the modified notation $L H_{+\times}, L H_{-\times}$and $L H_{\times \times}$.

The second class of cuts again corresponds to the situation when two final-state particles

are collinear. In this case the final-final state interference gives a large effect. We demand that all final-state particles are observable

$$
\angle\left(q_{1,2} p_{3,4}\right)>5^{\circ}, \quad \angle\left(q_{1,2} p_{4}^{\prime}\right)>5^{\circ}
$$



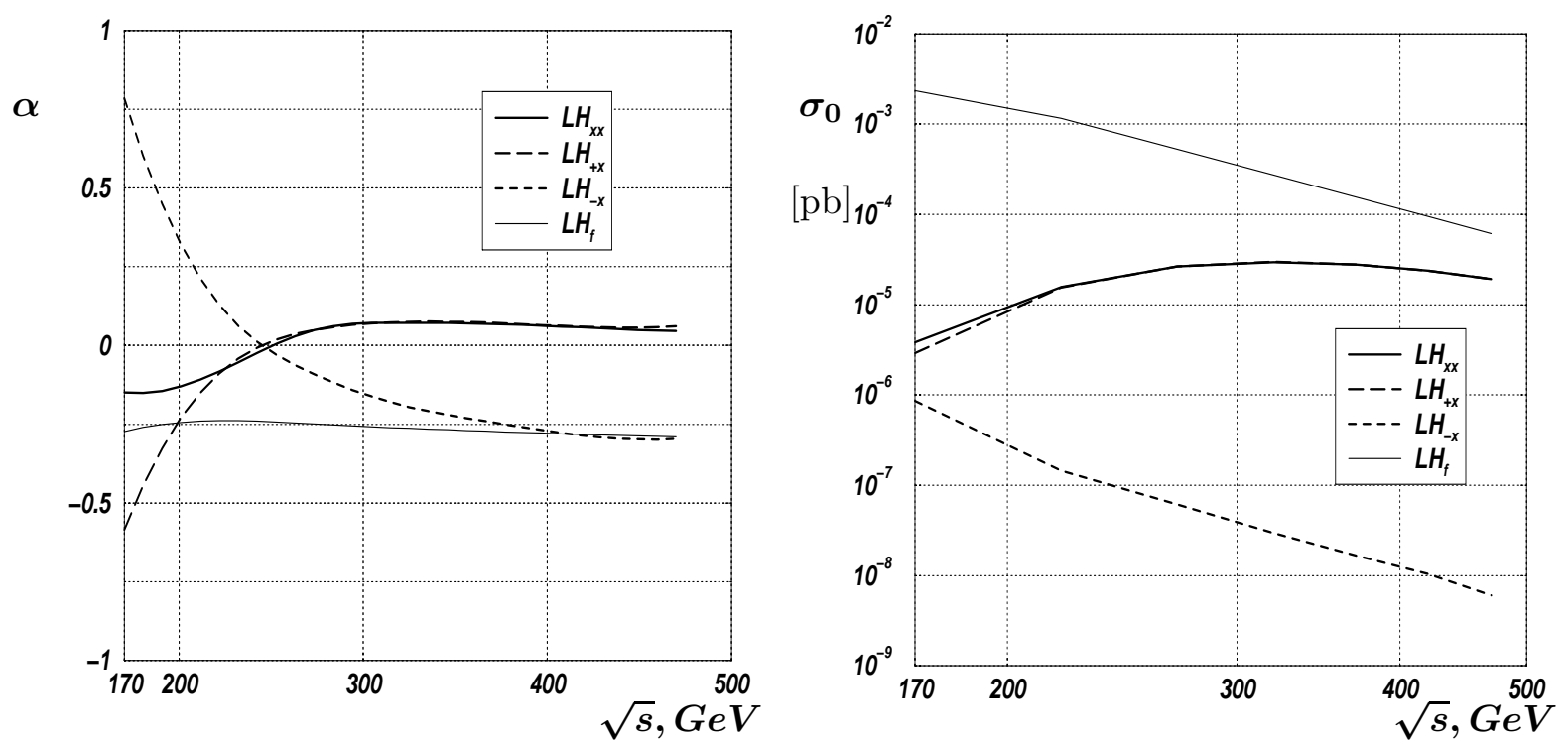

Figure 4: Ratio of the non-factorizable and factorizable parts of the photon radiation, $\alpha$, and the Born cross section, $\sigma_{\text {Born }}$, as a function of the CMS energy and the system of cuts. Leptonichadronic final states.

at least two final-state particles are quasi-collinear

$$
\angle\left(p_{3} p_{4}\right)<10^{\circ}, \quad \text { or } \angle\left(p_{3} p_{4}^{\prime}\right)<10^{\circ} \text {, }
$$

and the photon is far from all charged particles

$$
\angle\left(p_{3} k\right)>50^{\circ}, \quad L\left(p_{4} k\right)>50^{\circ}, \quad L\left(p_{4}^{\prime} k\right)>50^{\circ}, \quad \angle\left(q_{1,2} k\right)>50^{\circ} .
$$

Thus there is again only one possible choice

$$
L H_{\mathrm{f}} \text {. }
$$

We show in Fig. 4 the optimization parameters $\alpha(\sqrt{s}$, cuts $)$ and $\sigma_{\text {Born }}(\sqrt{s}$, cuts $)$ as functions of the CMS energy, $\sqrt{s}$, for all possible $L H$-cuts. Again, we see that the $L L_{-x}$-cut is the best in terms of the shape of the spectrum, but at the same time it is the worst in terms of statistics. $L H_{f}$ seems to be statistically the best overall throughout the energy region under consideration. From the point of view of the shape of the spectrum, $L H_{f}$ is not worse than any other system of cuts for higher energies. Note that for cuts restricting the jets to be quasi-collinear with the collider beams, the energy behaviour of the shape parameter $\alpha$ is more complicated than in the case of leptonic-leptonic final states. As mentioned above, if one of the quarks is quasi-collinear with the electron, the other one is automatically quasi-collinear with the positron. The effects coming from the two corresponding interferences have opposite signs. This can even lead to a change of sign of the combined effect at different collider energies. 


\subsection{Hadronic-hadronic final state}

Finally we consider the case when both $W$ bosons decay hadronically, with four jets present in the final state.

We again start from the case when the charged particles (i.e. jets) are collinear with the beam direction, with the photon well separated from the beam, $\angle\left(q_{1,2} k\right)>50^{\circ}$. The quarks with momenta $p_{3,4}$ and $p_{3,4}^{\prime}$ are required to be collinear with the initial leptons, $\angle\left(q_{1,2} p_{3,4}\right) \in\left(5^{\circ}, 20^{\circ}\right)$ and $\angle\left(q_{1,2} p_{3,4}^{\prime}\right) \in\left(5^{\circ}, 20^{\circ}\right)$. Since one cannot measure the charge of the jet experimentally. only the following combinations are available:

$$
H H_{22}, \quad H H_{13}, \quad H H_{\times \times},
$$

where the subscript denotes the number of jets that are collinear with the initial-state positron or electron. For example, $\mathrm{HH}_{13}$ means that there is one jet collinear with the positron, $q_{1}$, and three jets collinear with the electron, $q_{2}$. Again, the kinematics are such that at LEP2 energies not all of these cases are non-zero:

$$
H H_{13}=0 \text {, }
$$

and in fact only one case survives:

$$
H H_{22}=H H_{\times \times} .
$$

The second class of cuts corresponds to when two final-state particles (jets) are quasicollinear. In this case final-final state interference gives a large effect. We first demand that all final state particles are observable

$$
\angle\left(q_{1,2} p_{3,4}\right)>5^{\circ}, \quad \angle\left(q_{1,2} p_{3,4}^{\prime}\right)>5^{\circ},
$$

at least two final state particles are collinear

$$
\angle\left(p_{3} p_{4}\right)<10^{\circ}, \quad \text { or } \angle\left(p_{3} p_{4}^{\prime}\right)<10^{\circ}, \quad \text { or } \quad \angle\left(p_{3}^{\prime} p_{4}\right)<10^{\circ}, \quad \text { or } \quad \angle\left(p_{3}^{\prime} p_{4}^{\prime}\right)<10^{\circ} \text {, }
$$

and the photon is far from all of the charged particles

$$
\angle\left(p_{3,4} k\right)>50^{\circ}, \quad L\left(p_{3,4}^{\prime} k\right)>50^{\circ}, \quad L\left(q_{1,2} k\right)>50^{\circ} .
$$

There is again only one possible case:

$$
H H_{\mathrm{f}} .
$$

The optimization parameters $\alpha(\sqrt{s}$, cuts $)$ and $\sigma_{\text {Born }}(\sqrt{s}$, cuts $)$ are shown in Fig. 5 for the two possible cuts $H H_{\times \times}$and $H H_{f}$. As in the previous cases, $H H_{f}$ cut is better statistically, but $H H_{\times \times}$is better from the point of view of the shape of the photon spectrum. 

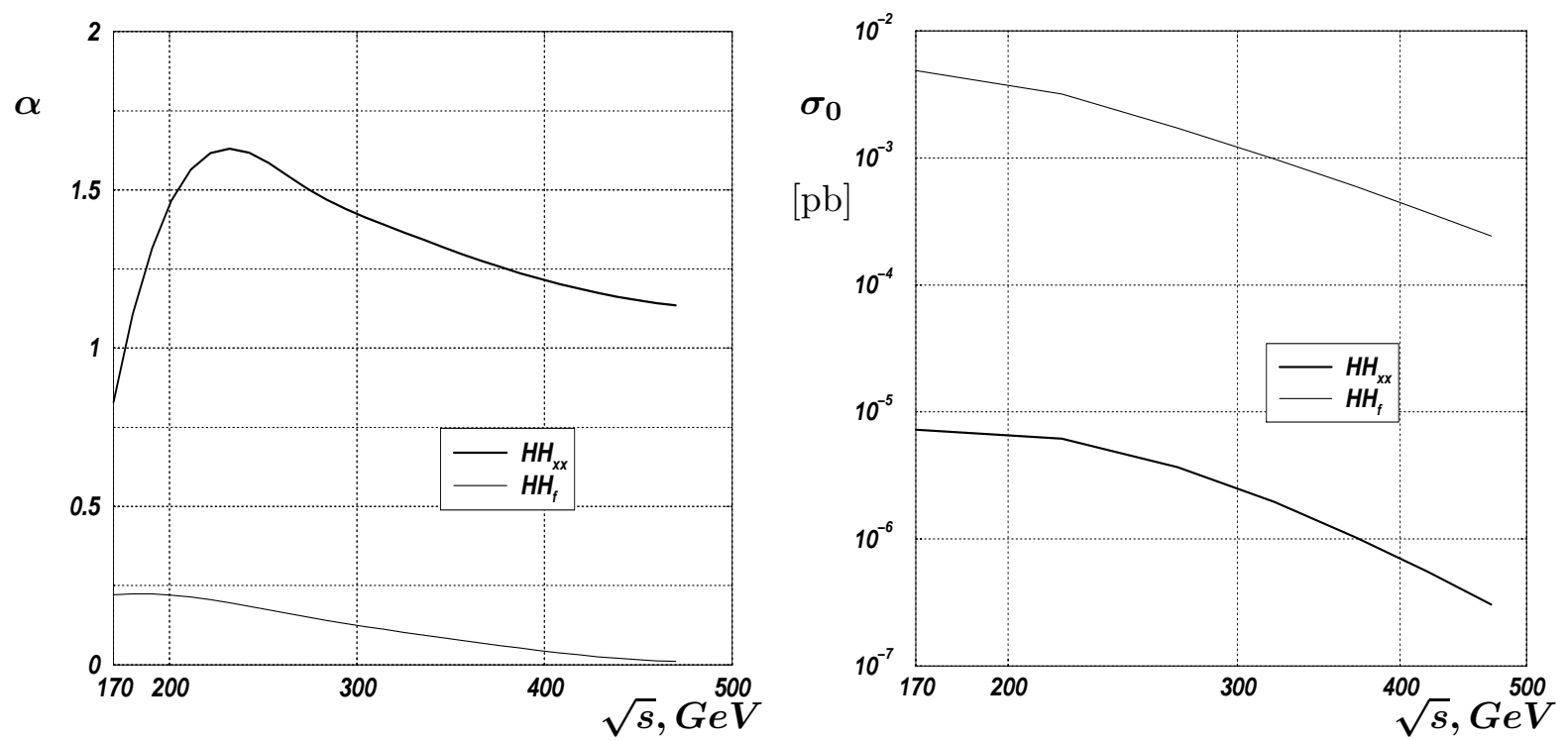

Figure 5: Ratio of the non-factorizable and factorizable parts of the photon radiation, $\alpha$, and the Born cross section, $\sigma_{\text {Born }}$, as a function of the CMS energy and the system of cuts. Hadronic-hadronic final states.

\subsection{Photon-photon colliders}

In recent years there has been a growing interest in high-energy photon colliders, using Compton back-scattering of laser light off the lepton beams at linear colliders to produce high-intensity, high-energy beams of photons, see e.g. Ref. [20]. Using $\gamma \gamma$ collisions to produce pairs of $W$ bosons offers certain advantages over the $e^{+} e^{-}$case. First, the cross section is an order of magnitude larger. Second, ISR effects are absent in this case and so kinematic reconstruction of the $W W$ final state is in principle more precise.

It is straightforward to extract the predictions for photon radiation in $\gamma \gamma \rightarrow W^{+} W^{-}$from our study of the more complicated $e^{+} e^{-}$case. In particular, our results for the final-final state interferences $L L_{\mathrm{f}}, L H_{\mathrm{f}}$ and $H H_{\mathrm{f}}$ can be applied directly to the $\gamma \gamma$ case. Moreover, as we have already explained, in the case of $W^{+} W^{-}$production in photon-photon collisions one can study observables integrated over the photon angle, to which factorizable corrections do not contribute, see Ref. [7].9] This enables us to utilize more events and makes studies in $\gamma \gamma$ collisions potentially more statistically powerful than in the $e^{+} e^{-}$-case.

\footnotetext{
${ }^{9}$ Note that there is a typo in Eq. (20) of the first reference in [7]. The normalization coefficients in front of the integrals in the first and second terms should be interchanged. The final result given by Eq. (22) is unchanged.
} 


\section{Concluding remarks}

A precision measurement of the total $W$ decay width presents a challenge for present and future experiments. Line-shape measurements are made difficult by the presence of neutrinos in the final state in the case of leptonic decay modes, and of hadronization corrections in the case of hadronic decays. The indirect measurement at hadron colliders, which uses the ratio of $W$ and $Z$ leptonic events, has an inherent uncertainty from parton distributions in the theoretical calculation of the total cross sections. It seems to be quite a challenging task to perform a precise direct measurement of the total $W$-width, independent of decay modes (and of the $Z$ measurements).

As discussed in Ref. [2], running a future linear collider in the 'LEP2' energy region may provide a unique opportunity for a high-precision measurement of the $W$ mass and width. The 'traditional' way of measuring $\Gamma_{W}$ is from a threshold scan of the total $W W$ cross section. Though statistically powerful, this method is not without problems. The uncertainties caused by beam-induced effects (beamsstrahlung, intrinsic energy spread, etc.) could be potentially

large. Moreover, the threshold strategy requires operating a linear collider at energy scan points well below threshold where the $W^{+} W^{-}$cross section is very small.

In this paper we have argued that the soft-photon radiation spectrum could also be used to obtain information on $\Gamma_{W}$. We emphasize that this is an independent method - in effect one is measuring the non-factorizable interference to the cross section, whose magnitude is controlled by the relative size of the photon energy and the $W$ width. The method is in principle very clean, requiring only a precise measurement of the soft (i.e. of order few $\mathrm{GeV}$ ) photon spectrum in $W^{+} W^{-} \gamma$ events. However, as we have seen, the effect in the inclusive distribution is very small and therefore is likely to be limited by statistics. On the other hand, we have shown that one can enhance the effect by employing angular cuts on the final-state particles. We have considered various different topologies and different $W$ decay channels. Both the sensitivity to the non-factorizable contributions and the overall number of events in the various channels are rather strongly dependent on the collision energy, and it should be possible to develop an optimal strategy given the parameters and running conditions of a future linear collider.

Our study necessarily falls short of any firm conclusion about the competitivity of our method, compared to the threshold scan for example, in determining $\Gamma_{W}$. At the very least, our method offers a complementary measurement, with completely different systematics. The next step would be to perform a detailed Monte Carlo study including detector and, where appropriate, hadronization effects. Among the questions that such a study could answer are: what is the efficiency for detecting very soft photons? can such photons be measured in the presence of hadronic jets? are the isolation and collinearity cuts we have used realistic? For a given collider energy it should be straightforward to estimate the number of soft photon events for each of the different topologies and decay channels, and by comparing this with the theoretical predictions, to estimate the statistical error on $\Gamma_{W}$. The results of our work suggest that a more detailed study is definitely worth pursuing. 


\section{Acknowledgements}

We thank T. Sjöstrand, L. Stodolsky and G. Wilson for useful discussions. VAK thanks the Leverhulme Trust for a Fellowship. The work of APC is supported in part by the UK Particle Physics and Astronomy Research Council. This work was also supported by EU Fourth Framework Programme "Training and Mobility of Researchers", contract FMRX-CT98-0194 (DG 12 - MIHT).

\section{References}

[1] J. Erler et al., hep-ph/0005024.

[2] G. Wilson, in Proceedings of the Linear Collider Workshop, Sitges 1999.

[3] T. Sjöstrand and V.A. Khoze, Z. Phys. C62 (1994) 281.

[4] M. Lancaster, hep-ex/9912031.

[5] T. Affolder et al. (CDF Collaboration), Fermilab-Pub-00/085-E, hep-ex/0004017.

[6] LEP Electroweak Working Group note, April 2000 (see www.cern.ch/LEPEWWG).

[7] Yu.L. Dokshitzer et al., Phys. Lett. B313 (1993) 171;

Yu.L. Dokshitzer et al., Nucl. Phys. B403 (1993) 65.

[8] V.A. Khoze, L.H. Orr and W.J. Stirling, Nucl. Phys. B378 (1992) 413.

[9] W. Beenakker, F.A. Berends and A.P. Chapovsky, Nucl. Phys. B548 (1999) 3.

[10] A. Denner, S. Dittmaier, M. Roth and D. Wackeroth, Phys. Lett. B475 (2000) 127.

[11] K. Melnikov and O. Yakovlev, Nucl. Phys. B471 (1996) 90;

W. Beenakker, A.P. Chapovsky and F.A. Berends, Phys. Lett. B411 (1997) 203; Nucl. Phys. B508 (1997) 17;

A. Denner, S. Dittmaier and M. Roth, Nucl. Phys. B519 (1998) 39; Phys. Lett. B429 (1998) 145 .

[12] R.M. Eisberg, D.R. Yennie and D.H. Wilkinson, Nucl. Phys. 18 (1960) 338.

[13] R.G. Stuart, Phys. Lett. B262 (1991) 113;

A. Aeppli, G.J. van Oldenborgh and D. Wyler, Nucl. Phys. B428 (1994) 126.

[14] V.S. Fadin, V.A. Khoze and A.D. Martin, Phys. Rev. D49 (1994) 2247.

[15] A.P. Chapovsky and V.A. Khoze, Eur. Phys. J. C9 (1999) 449.

[16] Yu.L. Dokshitzer, V.A. Khoze A.H. Mueller and S.I. Troyan, "Basics of Perturbative QCD”, Editions Frontiéres, Gif-sur-Yvette, 1991;

V.A. Khoze and W. Ochs, Int. J. Mod. Phys. A12 (1997) 2949. 
[17] A.E. Chudakov, Izv. Akad. Nauk SSSR, Ser. Fiz. 19 (1955) 650.

[18] F.E. Low, Phys. Rev. 110 (1958) 974;

T.H. Barnett and N.M. Kroll, Phys. Rev. Lett. 20 (1968) 86.

[19] Yu.L. Dokshitzer, V.A. Khoze and W.J. Stirling, Nucl. Phys. B428 (1994) 3.

[20] V.A. Telnov, hep-ex/0001029; hep-ex/0003024. 